\title{
Recursos educativos en museos online de arte contemporáneo. Tipología e implantación
}

\author{
Educational resources in online museums of \\ contemporary art. Typology and scope
}

\author{
NiCOLÁS DEL RÍO \\ Universidad de Santiago de Compostela, departamento de Historia del Arte, \\ investigador asociado. \\ josenicolas.delrio@usc.es
}

Recibido: 2 de abril de 2012

Aprobado: 30 de julio de 2012

\begin{abstract}
Resumen
El número de visitantes virtuales ha superado al de visitantes presenciales en numerosos museos. Como resultado, las instituciones museísticas se enfrentan al reto de atender al público no-presencial mediante el desarrollo y consolidación de servicios online. Entre ellos se encuentra uno de los cometidos fundamentales del museo: la educación. Esta investigación identifica y analiza los recursos educativos online presentes en 128 websites de museos de arte contemporáneo de todo el mundo para conocer su alcance y tipología. El análisis de los datos, realizado desde tres perspectivas, ofrece diversas conclusiones. Según la frecuencia de uso, los materiales más extendidos son de tipo editorial. Según el formato, son mayoritarios los modelos adaptados de la mediación presencial, aunque hay una presencia relevante de propuestas elaboradas a partir de las características de la Red. Según su interactividad y grado de participación, los formatos de la Web 2.0 son escasos y los ejemplos de colaboración y co-creación, anecdóticos. Adicionalmente, la investigación también aporta una perspectiva general sobre el apoyo desigual que los museos online de arte contemporáneo ofrecen a la educación formal y al aprendizaje informal. Palabras clave: museos online, visitantes virtuales, educación, arte contemporáneo.
\end{abstract}

Del Río, N. (2013): Recursos educativos en museos online de arte contemporáneo. Tipología e implantación. Arte, Individuo y Sociedad, 25(2) 233-245

\begin{abstract}
The number of virtual visitors has overtaken the number of physical visitors in numerous museums. Consequently, museum institutions face the challenge of catering to their virtual audience by developing and consolidating online services. Among these, there is an essential museum mission: education. The current research identifies and analyzes online educational resources in 128 contemporary art museums worldwide to gain insight into their typology and scope. The analysis of data, performed from three perspectives, offers several conclusions. According to the frequency of use, editorial content is most widespread. According to format, resources adapted from educational activities at the physical museum are more common, although there is a relevant number of materials based on online features. According to interactivity and participation, Web 2.0 formats are scarce and cases of collaboration and co-creation are minimal. Additionally, the analysis also provides an overview of the uneven support that online museums of contemporary art provide to formal education and informal learning.
\end{abstract}

Key Words: online museums, virtual visitors, education, contemporary art.

Del Río, N. (2013): Educational resources in online museums of contemporary art. Typology and scope. Arte, Individuo y Sociedad, 25(2) 233-245 
Sumario: 1. Introducción, 2. Metodología: selección y alcance de la muestra, 3. Resultados, 3.1. Implantación, 3.2. Formato, 3.3. Interactividad, 4. Discusión, 5. Conclusiones. Referencias.

\section{Introducción}

La ministra de cultura de España señalaba a finales de 2010: «los museos estatales tienen hoy más visitantes virtuales que presenciales y esas visitas virtuales se han incrementado un 23\% respecto al año anterior» (Garcia y Fraguas 2010). Esta tendencia, ya identificada en 2002 (Hawkey 2004:1), desborda el tradicional marco de acción del museo, centrado en atender a público local y turistas. El volumen de usuarios virtuales, la creciente duración de sus visitas a instituciones culturales (Rellie 2003:10) y sus expectativas de un acceso inmediato y ubicuo a contenidos museográficos digitales (Johnson, Adams \& Witchey 2011:4) ha impuesto a los museos la necesidad de desarrollar servicios online para sus visitantes no-presenciales.

Varios estudios realizados a lo largo de diez años sobre los intereses de los visitantes virtuales (Reynolds 1997; Kravchyna y Hastings 2002; CHIN 2006; Marty $\&$ Chen 2006) aportan una multiplicidad de perspectivas que convergen en cuatro áreas principales: información, para preparar la visita al museo físico o para decidir si merece la pena hacerla; trabajo, tanto profesional como académico; educación, como apoyo a la formación reglada o para el aprendizaje informal, y ocio, con propuestas que abarcan desde juegos para niños hasta compras online. Esta investigación busca profundizar en uno de estos cuatro ámbitos: la oferta educativa online y, en particular, la que proponen los museos de arte contemporáneo. El objetivo del estudio está encaminado a identificar y analizar sus recursos educativos en la Red para calibrar el grado de implantación que alcanza entre ellos la mediación online.

\section{Metodología: selección y alcance de la muestra}

Los datos que se exponen en este estudio han sido recopilados entre octubre de 2011 y enero de 2012 a partir de un corpus inicial de 324 museos online de arte contemporáneo de todo el mundo. La diversidad de idiomas presentaba en algunos casos una barrera para la consulta. A pesar de que la mayoría de websites dispone de una versión en inglés, habitualmente no abarca la totalidad de los contenidos y ofrece tan solo los más destacados. Para evitar posibles omisiones, estos museos se revisaron a través de «Google Translate» (http://translate.google.com).

El proceso de selección se realizó atendiendo a cinco criterios que delimitaron de forma progresiva la selección de referencias. El primer paso consistió en recoger únicamente websites de instituciones museísticas profesionales, desestimando las webs de museos que carecen de sede física y que existen únicamente en la Red. Este primer parámetro permitió descartar websites etiquetados con el engañoso término de «museo virtual». Una denominación demasiado vaga y especialmente problemática para las entidades profesionales, ya que otorga el rango de museo a cualquier colección online de objetos digitales aunque ésta haya sido creada por entidades o personas 
ajenas al ámbito museográfico. En segundo lugar, con el propósito de mantener la consistencia de las referencias a analizar, se excluyeron los museos que comparten fondos de arte contemporáneo con arte de otras épocas o de otras disciplinas. En tercer paso consistió en recopilar aquellas instituciones con website propio, es decir, cuyo URL o dirección de Internet identifica exclusivamente a un museo, no a una institución superior que informa sobre él. Siguiendo este criterio se rechazaron museos alojados dentro de portales turísticos, culturales, políticos y administrativos. Una vez identificadas las webs de instituciones museísticas profesionales con identidad y espacio online propios, se llevaron a cabo los dos últimos procesos de selección. La penúltima fase consistió en seleccionar las instituciones con contenidos educativos online. Para ello sólo se tuvieron en cuenta los websites que contaban con una sección etiquetada como "educación", "mediación", "pedagogía", "aprender", "acción cultural", etc. De este modo, la identificación de recursos online con valor educativo queda en manos de los propios museos, pues son ellos quienes deciden qué materiales cumplen esa función y merecen ser incluidos en dicho apartado. Para terminar el proceso de selección se aplicó un último criterio: excluir los museos que, a pesar de contar con una sección educativa que aloja materiales online, éstos sólo proporcionan información sobre actividades presenciales; es decir, calendarios de programación, formularios de reserva, memorias de actividades, consejos para visitas escolares, etc. Este proceso redujo el número inicial de referencias de 320 a 128.

\section{Resultados}

Una primera aproximación a los resultados permite identificar varias tendencias globales (ver Tabla 1). La primera de ellas ilustra el alcance de la oferta educativa online. Menos de la mitad de las entidades analizadas, el 40\%, dispone de recursos online, mientras que el $60 \%$ restante utiliza su web para dar a conocer la programación educativa en las salas y documentar acciones presenciales ya realizadas. Un segundo aspecto destacable, que afecta al $40 \%$ de museos que cuentan con materiales educativos online, es la polarización de éstos. Entre los diecisiete contenidos identificados, uno de ellos alcanza un $62,5 \%$ de implantación, mientras que ocho apenas superan el 3\%. Tomando estos extremos como referencia, es posible extraer una tercera valoración sobre los modelos educativos preferentes. Dieciséis de los diecisiete contenidos educativos son utilizados por casi un $18 \%$ de museos. Este dato revela que la amplia mayoría de museos - poco más del $82 \%$ - se inclina por ofrecer propuestas basadas en un único modelo. Un último aspecto global, revela que los museos de Europa y Estados Unidos son los principales proveedores de contenidos educativos en la Red ya que entre ambos suman el $80,31 \%$ (60,31\% y 20\%, respectivamente) de las referencias analizadas. La Tabla 2 presenta la distribución geográfica de los 320 museos analizados. 


\begin{tabular}{l|l|l|c} 
& & \multicolumn{1}{|c}{ CONTENIDOS EDUCATIVOS } & \% \\
\hline Más de 20\% & 1. & Guías / Cuadernos / Fichas para público escolar & 62,5 \\
\hline Entre 10\% y 20\% & 2. & Audios & 17,96 \\
\hline & 3. & MicroWebs educativas & 18,75 \\
\hline & 4. & Vídeos & 16,62 \\
\hline Entre 5\% y 10\% & 5. & Blogs & 10,15 \\
\hline & 6. & Guías (sin especificar público) & 9,37 \\
\hline & 7. & Interactivos / Exposiciones online & 7,81 \\
\hline & 8. & Enlaces a la colección & 7,03 \\
\hline & 9. & Enlaces a exposiciones & 7,03 \\
\hline & 10. & Artículos / Ensayos / Reseñas / Críticas & 3,12 \\
\hline & 11. & Catálogos de referencias bibliográficas & 3,12 \\
\hline & 12. & Cursos Online & 2,34 \\
\hline & 13. & Revistas & 1,56 \\
\hline & 14. & Glosarios & 1,56 \\
\hline & 15. & Foros & 1,56 \\
\hline & 16. & Wikis & 0,78 \\
\hline & 17. & «Mash-ups» & 0,78 \\
\hline & & & \\
\hline
\end{tabular}

Tabla.1. Contenidos educativos online presentes en las secciones educativas de 128 museos online de arte contemporáneo. (Fuente: elaboración propia).

Los datos recogidos en la Tabla 1 favorecen un análisis desde tres perspectivas: su grado de implantación: ¿qué contenidos son los más -y menos- utilizados?, su formato: ¿provienen de la mediación presencial o aprovechan las características de la Red? y su interacción con los visitantes: ¿son propuestas cerradas del museo o permiten la participación de los visitantes?

\begin{tabular}{l|c|c}
\hline Distribución geográfica & Númerode museos & Con contenidoseducativos online \\
\hline Europa Occidental & 193 & 74 \\
\hline Europa del Este y Rusia & 15 & 1 \\
\hline Oriente Próximo y Medio & 4 & 0 \\
\hline Asia & 10 & 2 \\
\hline Oceanía & 14 & 8 \\
\hline Africa & 0 & 0 \\
\hline Norteamérica & 64 & 40 \\
\hline Centroamérica y Caribe & 3 & 0 \\
\hline Sudamérica & 17 & 3 \\
\hline
\end{tabular}

Tabla.2. Distribución geográfica de museos analizados. 


\subsection{Implantación}

El contenido educativo preferente, a gran distancia de los dieciséis materiales restantes -que apenas alcanzan el 20\%-corresponde a guías docentes, cuadernos para alumnos, fichas y dossiers didácticos. Se trata de un conjunto de materiales que han sido desarrollados para servir de apoyo a alumnos y profesores de centros de formación reglada. La adecuación entre estos contenidos y sus destinatarios académicos queda de manifiesto en una presentación online que habitualmente se desglosa por niveles escolares (infantil, primaria y secundaria), por tipo de destinatarios: docentes y/o alumnos (en algunos casos, los contenidos de educación infantil también se sugieren para un público familiar) o muy ocasionalmente por asignaturas. Una ausencia reseñable dentro este grupo es la enseñanza universitaria; cuando se hace referencia ella es siempre en relación al museo físico: visitas guiadas, ciclos de conferencias, etc.

Con una implantación entre el 10\% y 20\%, se encuentran contenidos audiovisuales, microwebs educativas y blogs. Los contenidos realizados en vídeo, en el 55\% de los casos, están incrustados en la web del museo desde repositorios multimedia externos, donde realmente residen. Entre los museos analizados, el más popular es «YouTube», seguido de «Vimeo»y «Artbabble», éste último creado específicamente para museos. Haciendo click sobre un vídeo, se accede a la web del repositorio audiovisual y, una vez allí, el usuario puede escribir comentarios, responder a los ya existentes y visualizar otros vídeos del canal del museo o de temática relacionada. Sin embargo, esta opción de participación de los usuarios no existe dentro de la propia web del museo. Los audios, también conocidos como «podcasts», se alojan dentro de las propias webs de museos en un $78,26 \%$ de casos, posiblemente debido al menor peso de los archivos. Sólo de forma minoritaria (8,6\%) se utiliza «iTunes» como repositorio externo. A este respecto, es necesario señalar que algunos museos tienden a reunir todos sus recursos audiovisuales en una sección específica-fuera de la sección educativa- que adopta la forma de un canal temático, como por ejemplo, «Tate Channel» o «Media Zone», éste último perteneciente a la KunstHalle Wien. A pesar de que este tipo de repositorios pueden llegar a ser bastante amplios, en pocos casos (un 7,03\%) se aprovechan apara enlazarlos desde la sección educativa. Por su parte, las micro-webs concentran todos los contenidos educativos del museo en un único espacio que, de este modo, adquiere una entidad propia. De ahí que algunas de ellas adopten un nombre y una dirección únicos (redstudio.moma.org, mnacedu-ca. org, etc.) siendo, en algunos casos, indicativos del público al que están destinadas (por ejemplo, junior.centrepompidou.fr o icateens.org, ambas diseñadas para adolescentes). Con respecto a los blogs, un $38,46 \%$ de ellos están dedicados exclusivamente a educación pero no como herramienta de mediación, sino como un sistema de notificación informativa o de archivo documental.

Con valores entre el 5\% y el 10\% se encuentran guías, interactivos y exposiciones online, junto con enlaces a la colección online y a información sobre exposiciones físicas. Las guías son formatos análogos a los materiales escolares descritos en el primer grupo, con la diferencia de que no tienen un planteamiento para el aula ni han sido adscritos a ningún público académico. Al no estar específicamente enfocadas a este grupo de visitantes, funcionan como recursos para el aprendizaje informal de 
visitantes individuales. En algunos casos están específicamente dirigidos a adultos, mientras que en otros no se menciona el destinatario y, por tanto, se asume un público general. A pesar de que carecen de un nombre genérico, algunos museos las identifican como «guías de reflexión», «guías de lectura», «dossiers didácticos», «guías de exposición», etc. Esta miscelánea de nombres son indicativos de su variada función: preparar una visita a una exposición, reflexionar posteriormente sobre ella o profundizar sobre determinadas obras y artistas. Con respecto a los módulos interactivos y las exposiciones online, ambos se han agrupado en una única categoría, atendiendo a su similitud formal y de contenido, y siguiendo las pautas que marcan los propios museos que, en vez de identificarlos como propuestas separadas, los agrupan bajo la etiqueta común de «interactivos» o «piezas interactivas». Se caracterizan por presentar una aproximación formal muy variada, por tratar contenidos monográficos de una forma más generalista que experta, por incorporar una combinación de diferentes recursos audiovisuales y por cierto planteamiento lúdico que reclama la interacción del usuario. El desarrollo de este tipo de recursos se encuentra poco extendido, limitándose a los grandes museos anglosajones. De las 10 instituciones que disponen de ellos, sólo las norteamericanas tienen más de tres. En lo que respecta a los enlaces a la colección online, resulta especialmente llamativo que sólo 9 museos planteen sus fondos online como recurso educativo. Fuera de este porcentaje, las colecciones online no aportan enlaces a propuestas de mediación ni a otros recursos que ayuden a profundizar en una pieza, un creador o un contexto.

El cuarto bloque de contenidos educativos está compuesto por una amplia miscelánea de recursos cuya incidencia apenas supera el 3\%. Dos tipos de estos materiales pueden englobarse bajo la categoría de publicaciones (artículos, ensayos, reseñas y críticas; y revistas) cuyo valor educativo reconocen 4 museos. La consulta de referencias bibliográficas del centro de documentación del museo también es una opción poco frecuente aunque resulta especialmente valiosa para un público experto, posiblemente investigadores, profesionales o profesores y estudiantes universitarios. Los cursos online representan opciones de formación fuera del ámbito de la educación académica mientras que los glosarios funcionan como material de referencia y suponen un refuerzo de aprendizaje. Por último, foros y wikis y «mash-ups» (es decir, la re-elaboración de recursos procedentes de diversas fuentes para crear un producto nuevo) representan, junto con blogs y repositorios audiovisuales, la incursión de los museos en la Web 2.0. Sin embargo, los foros y wikis sólo se encuentran en dos sedes del mismo museo, el Guggenheim de Nueva York y Bilbao, y el único ejemplo de «mash-up» está integrado en «Arts ConnectEd», una web educativa que el Walker Art Center de Minneapolis comparte con el Minneapolis Institute of Arts.

\subsection{Formato}

Atendiendo al formato, 10 de las 17 propuestas educativas reflejan modelos y planteamientos heredados de la mediación presencial, marcando una línea continuista basada en la adaptación y trasvase al medio online. Así, los cuadernos online son comparables -cuando no idénticos- a los utilizados durante visitas escolares; las guías y fichas contienen información similar a la que se ofrece en hojas de sala, textos de paneles y cartelas. Los artículos, ensayos y reseñas son materiales expertos 
análogos a las publicaciones que se encuentran a la venta en la tienda y a artículos publicados en revistas profesionales. La consulta de la base de datos bibliográfica del museo a través de la Red produce, presumiblemente, idénticos resultados a la consulta «in situ». Los interactivos online adaptan el formato y planteamiento de los puestos de consulta instalados en algunos museos. Los audios pueden considerarse una actualización de las tradicionales audioguías mientras que los vídeos también trasladan a la Red un recurso del lenguaje expositivo presencial. Por contraste, las micro-webs educativas, colecciones online y repositorios audiovisuales, junto con blogs, foros, wikis y «mash-ups», son modelos desarrollados a partir de la estructura hipermedia de la Red, que permite la consulta de fondos museográficos de una forma diferente a los itinerarios que marcan las salas o que proponen los recursos tradicionales de mediación.

\subsection{Interactividad}

La diferenciación entre los formatos tradicionales de la mediación presencial y las propuestas creadas para el medio online implica también diferentes modelos de interactividad. Los primeros tienen carácter estático mientras que los segundos se basan interacción y participación. Los contenidos estáticos han sido concebidos para la consulta, ya sea mediante lectura, audición o visionado. Se trata de materiales «cerrados» que presentan el discurso del museo sin posibilidad de alteración. Los módulos interactivos, las bases de datos bibliográficas y las colecciones online también proponen contenidos cerrados pero organizados en una estructura abierta y flexible que permite a los usuarios la posibilidad de procurarse la información que les resulte más significativa sin seguir obligatoriamente una secuencia predeterminada. Finalmente, los blogs, foros, wikis y «mash-ups»-incluso audios y vídeos, si se ven desde los repositorios audiovisuales- permiten participar al público, valorando los contenidos de otros y aportando los suyos propios a un fondo de conocimiento común.

\section{Discusión}

Los datos sobre contenidos y formatos online tienen una rápida caducidad. Su verdadero valor reside en un seguimiento continuado que permita contrastar su evolución o estancamiento. En consecuencia, los resultados de esta investigación se analizan con respecto a varios estudios previos, al tiempo que se ofrecen como referencia para investigaciones futuras. Cuatro investigaciones anteriores sobre materiales educativos en museos online sirven de base para valorar este estudio. Es necesario advertir, sin embargo, que el ámbito de dichas investigaciones no se ocupa específicamente de museos de arte contemporáneo y que sus referencias abarcan zonas geográficas diversas. Por ello, los datos citados deben considerarse como un marco de referencia, más que valores específicos de contraste.

Una primera investigación sobre materiales educativos, tanto presenciales como online, entre museos de arte norteamericanos indica que un 54,1\% de ellos ofrecen actividades educativas online (Wetterlund y Sayre 2003:10); una actualización del mismo estudio realizada seis años más tarde elevaba ese porcentaje a 64\% (Wetterlund 
y Sayre 2009:21). Entre los museos de arte contemporáneo, resulta notable que la mediación online presente una cifra considerablemente menor y no supere el $40 \%$ (128 de 320 museos). Esto puede deberse a que los museos valoran que el impacto de una obra auténtica en el entorno físico no es comparable con la visualización de un sustituto digital y que, por ello, las acciones educativas son preferibles «en los lugares en los que se produce el hecho artístico» (del Río 2007:106). No obstante, la Red Canadiense de Información sobre el Patrimonio (CHIN) en su estudio sobre «La Calidad de la Experiencia Online de los Usuarios de Museos» (Soren 2004) también atribuye a la visita online -cuando está planteada en términos de interactividad y descubrimiento- la capacidad de proporcionar «un rango de emociones en respuesta a la belleza, a una atmósfera evocadora, a nuevos aprendizajes e interactividad». Para ello, el lenguaje audiovisual, hipermedia e interactivo no debe quedarse en una fase meramente contemplativa, sino «estar inmerso en la interacción comunicativa que le añade significación» (Santibáñez 2006:157). Sin embargo, cuando las experiencias online se plantean como «museografía estática» (Santacana y Hernández 2006: 262263), es decir, como contenidos mostrados de forma pasiva a un público concebido solamente como receptor, su impronta en el visitante queda mermada.

Esta preocupación queda patente en un segundo sondeo sobre materiales educativos online realizado por Varisco y Cates (2005). Aunque los autores reconocían el valor de los materiales estáticos como recursos que facilitan la interpretación de colecciones y exposiciones, criticaban la pobreza, e incluso la ausencia, de herramientas de colaboración social, de más recursos de audio y vídeo y de instrucción online. Los datos recogidos en el presente estudio muestran que los museos sí han adoptado una variedad considerable de recursos online, sin embargo, de los 17 tipos identificados, 16 tienen una incidencia inferior al 18\%. El recurso preferente (con un 62,5\%) corresponde a archivos de tipo editorial que contienen textos e imágenes en un formato cerrado y carente de interactividad (habitualmente pdfs y en un número anecdótico de casos Word y PowerPoint). Aunque existen repositorios externos como «Isuu» o «SlideShare» para compartir este tipo de archivos, sólo tres museos hacen uso de ellos. Una posible explicación de su gran popularidad es que se trata de materiales que habían sido previamente desarrollados en soporte informático para acciones educativas presenciales o para publicaciones y que, una vez maquetados, su conversión a los formatos anteriores resulta inmediata. La proliferación de vídeos y audios se ha visto ayudada, sin duda, por «YouTube» e «iTunes», que han contribuido a reducir los problemas asociados al alojamiento y transferencia de archivos. No obstante, otros formatos 2.0 siguen escasamente representados. Sólo los blogs sobrepasan el $10 \%$, mientras que foros, wikis y «mash-ups» figuran cada uno en un único museo. Los interactivos, otros contenidos que requieren la participación de los usuarios, apenas suponen el $8 \%$, aunque en este caso sería necesario valorar individualmente el alcance de su interactividad ya que con frecuencia, en vez de animar al aprendizaje, se reducen a propuestas que perpetúan la pasividad, invitando a los usuarios «a pulsar y hacer clic» (Marstine 2006:227). La proliferación de archivos estáticos contribuye a forjar una percepción del museo online como espacio de almacenamiento. Baste recordar que 10 de los 17 contenidos educativos identificados responden a esta tipología y que el modelo más extendido se basa en un formato de descarga e impresión. 
En los museos analizados, las secciones educativas de las webs tienden a convertirse en bibliotecas digitales que reúnen listas de enlaces a archivos organizados por formato o fecha. Este sistema se encuentra más próximo a los sistemas de catalogación interna del museo que a acciones de mediación. A este respecto, Anderson (1999:57) ya mencionaba en los primeros años de la Red la necesidad de desarrollar proyectos educativos online marcados «por las necesidades del público, no por las de los sistemas de gestión de las colecciones ni de los datos contenidos en ellas».

Un tercer estudio, realizado por Bearman y Trant (2005) está enfocado hacia los públicos a quienes van dirigidos los materiales educativos online. Sus conclusiones indican que en el $66 \%$ de los museos la mediación online está concebida para un público general, que un $46 \%$ de ellos también dispone de una oferta para niños y que más de un tercio (sin especificar numéricamente) tiene propuestas para estudiantes de secundaria y visitantes presenciales. Sorprendentemente, los estudiantes y docentes de educación superior apenas está representados (sin especificar numéricamente), ni siquiera entre los propios museos universitarios, de los cuales sólo un $22 \%$ contempla contenidos para este público. Esta información invita a una doble apreciación de los datos recogidos sobre museos de arte contemporáneo.

Cuantitativamente, el destinatario principal de las propuestas de los museos analizados es el público perteneciente a instituciones académicas de infantil, primaria y secundaria. Los cuadernos, guías, fichas y dossiers didácticos multiplican más de 3 veces y medio la incidencia de cualquier otro formato educativo. Una única wiki destinada a docentes y las micro-webs educativas completan el conjunto de recursos específicamente destinados a la educación formal. A pesar de que los recursos de apoyo a alumnos y docentes son los más relevantes en número, utilizan un número mínimo de formatos (aunque las micro-webs contribuyen a ampliar ligeramente este rango ya que incluyen con frecuencia piezas audiovisuales, interactivos y blogs).

La distinción entre público general y público de instituciones académicas presenta una particularidad adicional. Mientras que los visitantes integrados en la educación formal disponen de materiales adaptados a diferentes niveles académicos (y por tanto de conocimientos), los visitantes que se encuentran fuera de estos grupos no reciben una atención igualmente especializada. Para la mediación online el público general es considerado por el museo como un grupo falsamente homogéneo, «como si todos los visitantes tuvieran la misma formación cultural y compartieran intereses similares» (Checa, 2008). A pesar de que la visita al museo como experiencia de aprendizaje se encuentra mucho más cerca «del aprendizaje del entorno que del proceso enseñanza/ aprendizaje de las escuelas» (Lee 1968:373), los recursos online diseñados para el aprendizaje informal (es decir, los no específicamente adscritos a la educación formal) utilizan una amplia variedad de formatos pero tienen un incidencia tres veces menor a las propuestas diseñadas para la formación reglada.

Una última apreciación está relacionada con la presencia de blogs, foros, wikis, «mash-ups»y repositorios compartidos, todos ellos identificados como tecnologías pertenecientes a la Web 2.0 (Anderson 2007:7-13, Espadas 2009:7-9). Estos recursos facilitan a los visitantes un papel activo que marca un punto de inflexión en la relación entre público y museo. Permiten ensanchar la actividad individual de consulta para dar cabida a la posibilidad de colaboración con la institución y de compartir con 
otros visitantes. Un cuarto estudio de 2010 (López, Margapoti, Maragliano y Bove) proporciona datos de contraste adicionales sobre el uso de los formatos 2.0 en 240 museos de Inglaterra, Francia, España, Italia y Estados Unidos. A pesar de que esta investigación no está acotada únicamente a materiales educativos, las cifras no son especialmente divergentes aunque sí ligeramente superiores. Al igual que en el presente estudio, los blogs son los medios sociales más populares, con porcentajes que oscilan entre el 16,7\% y el 2,1\% (Inglaterra y España, respectivamente); mientras que los aquí analizados alcanzan un 10,5\%. El conjunto de foros, wikis y «mash-ups» presenta también los porcentajes más bajo de incidencia, oscilando entre un $2,1 \%$ y un $4,2 \%$. Por contraste, estas cifras duplican holgadamente las de esta investigación, que muestran porcentajes entre el $0,78 \%$ y el $1,58 \%$.

Sin embargo, las fórmulas 2.0 en los museos analizados no se integran efectivamente en la web institucional -con la excepción de los blogs- sino que utilizan repositorios externos o formatos paralelos; posiblemente con la intención de establecer una división clara entre los contenidos institucionales y los contribuidos por los visitantes. A este respecto, Crenn y Vidal (2008) sostienen que la participación de la audiencia significa un reto para la autoridad del museo ya que «las aportaciones de los visitantes suponen, en último término, una injerencia en el control de la información». De hecho, las propuestas que facilitan una mayor colaboración de los visitantes son las menos implantadas. Los foros, wikis y «mash-ups» no superan el $1,56 \%$; los blogs, aunque tienen una repercusión unas 6 veces más alta, muestran cierta desvirtuación puesto que, al menos en relación con contenidos educativos en museos de arte contemporáneo, tienden a funcionar como agendas, apoyos promocionales o archivos de actividades pasadas. La baja presencia de medios sociales para propósitos educativos contrasta con las previsiones para los próximos 5 años del «Horizon Report, Museum Edition» (2011:4-5) cuyo objetivo consiste en «identificar tendencias que afectan a la práctica educativa y de interpretación en museos». Entre las seis consideraciones más relevantes que detalla el informe figura «un creciente coro de voces que abogan por un papel más activo de los visitantes a la hora de dar forma a las actividades de los museos».

\section{Conclusiones}

El crecimiento continuado de visitantes online impone a los museos la necesidad de ofrecer en su web no sólo información sino también servicios, entre ellos la mediación. A juzgar por los museos de arte contemporáneo analizados en este estudio, el cometido educativo se realiza fundamentalmente trasvasando contenidos de tipo editorial, similares a los usados tradicionalmente para el público presencial. Este tipo de materiales son estáticos y fomentan la idea del museo online como un repositorio de archivos digitales. La presencia de contenidos audiovisuales y colecciones online no contribuye a mejorar esta percepción ya que habitualmente se presentan en secciones autónomas que funcionan como bibliotecas de recursos organizadas por parámetros similares a los utilizados en la gestión y catalogación internas. Comparativamente, los materiales educativos concebidos para la Red, aunque son variados, tienen una baja implantación y apenas hacen uso de los formatos de la Web 2.0. 
El público principal de los materiales educativos son docentes y estudiantes pertenecientes a la educación formal aunque con una llamativa ausencia de propuestas para la educación superior. El aprendizaje informal de adultos se plantea para un público general falsamente homogéneo, sin una segmentación por nivel de conocimientos o intereses. En general, los contenidos educativos online en los museos de arte contemporáneo analizados se perciben como un servicio especializado para centros académicos y guardan poca relación con otros contenidos online que faciliten la exploración y el descubrimiento individual. Las facetas multimedia, interactiva y colaborativa del espacio online son poco utilizadas en favor de una museología más estática.

El uso de la web del museo como una plataforma activa de conocimiento sólo parece evidente para un grupo reducido de museos cuyos websites diferencian entre «aprender en el museo» y «aprender en la web». Al reconocer la especificidad de cada ámbito, se hace más evidente la necesidad de una mediación diferenciada pero, sobre todo, resulta más sencillo reconocer las características propias de cada entorno y aplicarlas adecuadamente a propuestas didácticas

\section{Referencias}

Anderson, P. (2007). What is Web 2.0? Ideas, technologies and implications for education. JISC Technology and Standards Watch. Febrero 2007. Bristol: JISC. Disponible en http://www.jisc.ac.uk/media/documents/techwatch/tsw0701b.pdf. Consultado el 1-10-2011.

Anderson, D. (1999). A Common Wealth: Museums in the Learning Age. London: Department of Culture, Media and Sport.

Bearman, D. y Trant, J. (2005). Survey of Museum Web Implementations. Archives \& Museum Informatics. Disponible en http://www.archimuse.com/research/ mwbenchmarks/report/mwbenchmarks2005.html. Consultado el 6-11-2011

Checa, F. (2008). Crisis y final de una idea del museo. Revista de Libros 133, 3-7. Disponible en http://www.revistadelibros.com/articulos/crisis-y-final-de-unaidea-del-museo. Consultado el 24-11-2011.

C.H.I.N. (2006). 2004 Survey of Visitors to Museums' Web Space and Physical Space. Quebec, Canada: Canadian Heritage Information Network. Disponible en http://www.pro.rcip-chin.gc.ca/contenu_numerique-digital content/2004survey-2004survey/index-eng.jsp. Consultado el 6-11-2011.

Crenn, G. \& Vidal, G. (2008). L'introduction des technologies web 2.0 dans les musées d'artmoderne et contemporain: vers de nouvelles relations entre l'institution muséale et sespublics? Musée 2.0, 1 de abril. Centre Pompidou, Paris. Disponible en http://www.centrepompidou.fr/musee2.0/Crenn_Vidal. html. Consultado el 8-12-2011.

Espadas, J. (2009). Museums Web 2.0 Ranking. Dpto. Sistemas de Información, Fundación Colección Thyssen-Bornemisza, Madrid. Disponible en http://blogs. museothyssen.org/tecnologico/wp-content/uploads/2009/06/Museum_research_ es.pdf. Consultado el 9-11-2011. 
Del Río, V. (2007). Educación y arte contemporáneo como prácticas. El área de educación en el Museo Patio Herreriano. In Fernández, O. \& del Río, V. (Eds.), Estrategias críticas para una práctica educativa en el arte contemporáneo (106-113). Valladolid: Museo Patio Herreriano.

García, R. y Fraguas, A. (2010, noviembre 24). «Minidebate» en el Congreso sobre el estado de la Cultura. El País. Disponible en http://www.elpais.com/articulo/ cultura/Minidebate/Congreso/estado/Cultura/elpepucul/20101124elpepucul_4/ Tes. Consultado el 4-1-2012.

Hawkey, R. (2004). Learning with Digital Technologies in Museums, Science Centres and Galleries: report 9. King's College, London. Bristol, UK: Futurelab. Disponible en http://www2.futurelab.org.uk/resources/documents/ lit_reviews/Museums_Galleries_Review.pdf. Consultado el 4-1-2012

Kravchyna, V. y Hastings, S. K. (2002). Informational Value of Museum Web Sites. First Monday 7 (2). Disponible en http://firstmonday.org/htbin/cgiwrap/bin/ojs/ index.php/fm/ article/view/929/851. Consultado el 17-12-2011.

Johnson, L., Adams, S., y Witchey, H. (2011). The NMC Horizon Report: 2011 Museum Edition. Austin, Texas: The New Media Consortium.

Lee, R. (1968). The Future of the Museum as a Learning Environment. Computers and their Potential Applications in Museums, 15-17 de abril. Metropolitan Museum of Art, New York.

Marstine, J. (Ed.) (2006). New museum theory and practice. Oxford, Inglaterra: Blackwell Publishing.

López, X., I. Margapoti, R. Maragliano \& G. Bove (2010). "The presence of Web 2.0 tools on museum websites: a comparative study between England, France, Spain, Italy and the USA". Museum Management and Curatorship, 25, 235-249.

Marty, P. F. y Chen, H. (2006). The Digital Museum in the Life of the User. Proceedings of the American Society for Information Science and Technology 42. doi: $10.1002 /$ meet. 1450420132

Rellie, J. (2003) Tate Online. Towards a third generation museum website. Proceedings of ICHIM03, 8-13 de Abril. École du Louvre, Paris. Disponible en http://www.archimuse.com/publishing/ichim03/080C.pdf. Consultado el 1512-2011.

Reynolds, R. (1997). Museums and the Internet: What purpose should the information supplied by museums on the World Wide Web serve?. (Tesis no publicada). Department of Museum Studies, Leicester University, United

Kingdom.Santacana, J. y Hernández, F. X. (2006). Museología Crítica. Gijón:

Trea.Santibánez, J. (2006). Los museos virtuales como recurso de enseñanzaaprendizaje. Comunicar 27, 155-162. Disponible en http://www.revistacomunicar. com/verpdf.php?numero=27\&articulo=27-2006-24. Consultado el 29/11/2011).

Soren, B. (2004). Research on 'Quality' in Online Experiences for Museum Users. Quebec, Canada: Canadian Heritage Information Network. Disponible en http:// www.pro.rcip-chin.gc.ca/contenu_numerique-digital_content/recherche_qualiteresearch_quality/index-eng.jsp. Consultado el 23-11-2011. 
Varisco R. y Cates, W. (2005). Survey of Web-based educational resources in selected U.S. art museums. First Monday 10 (7). Disponible en http:// firstmonday.org/htbin/cgiwrap/bin/ojs/index.php/fm/article/view/1261/1181. Consultado el 18-12-2011.

Wetterlund, K. y Sayre, S. (2003). Art Museum Education Programs Survey. Museum-Ed. Disponible en http://www.museum-ed.org/index.php?option=com content\&view $=$ article \&id=63:2003. Consultado el 16-12-2011.

Wetterlund, K. y Sayre, S. (2009). 2009 Art Museum Education Programs Survey. Museum-Ed. Disponible en http://www.museum-ed.org/index.php?option=com content\&view=article\&id=118:2009. Consultado el 16-12-2011. 